\title{
El encuadre de las enfermedades raras en los medios de comunicación españoles
}

\section{The framing of rare diseases in the Spanish media}

\author{
Paloma López-Villafranca*, Antonio Castillo-Esparcia** \\ *University of Málaga, Andalucía-Tech, Spain \\ **University of Málaga, Andalucía-Tech, Spain
}

Abstract

\begin{abstract}
El colectivo de pacientes con enfermedades raras en España ha destinado sus esfuerzos en los últimos cinco años en lograr mayor visibilidad en los medios de comunicación. Con patologías que afectan a menos de 5 por cada 10.000 habitantes, se enfrentan al reto de ocupar un lugar en la sociedad como colectivo para legitimar sus propuestas y sensibilizar a la opinión pública. En este artículo analizamos 527 piezas que se han publicado en prensa, (El País, El Mundo, ABC, La Vanguardia, El Periódico), que se han emitido en radio (SER, COPE, Onda Cero, RNE), y televisión (TVE, Telecinco, Antena 3, La Sexta, Cuatro), en el periodo de 2012 a 2014. Los resultados muestran que ha aumentado la visibilidad y el conocimiento sobre estas patologías y se refleja una imagen proactiva de este colectivo gracias a sus iniciativas y reivindicaciones.
\end{abstract}

Palabras Claves: enfermedades raras; medios de comunicación; España; encuadre, tratamiento mediático.

Abstract

Patients with rare diseases have aimed their efforts in the last five years to achieve greater visibility in the media and get funds to suplir the research of their treatmens. For these patients, with diseases affecting less than 5 per 10,000 of the population, it is a challenge to take their place in society as a group to legitimize their proposals and make aware the public opinion. We have analyzed 527 pieces in press (El País, EI Mundo, ABC, La Vanguardia, El Periódico), radio (Cadena Ser, Onda Cero, Cope and RNE) and television ( Telecinco, Antena 3, La 1, La Sexta, Cuatro), from 2012-2014. The results show that it has increased the visibility and the knowledge of these diseases and the image of this group has improved because of their initiatives and demands.

Keywords: rare diseases; media; Spain; framing, media treatment.

\section{Introducción}

En los últimos cinco años el colectivo de pacientes con enfermedades raras ha dirigido sus acciones y estrategias a aparecer en los medios de comunicación tradicionales para hacer visibles estas patologías. Un objetivo prioritario desde que en 2013 las organizaciones de pacientes y el gobierno español acordaran que éste sería el año destinado a las enfermedades poco frecuentes o denominadas raras. Para que estas patologías tengan tal condición, según Posada (2008: 9), tiene que darse el caso de tener una prevalencia "inferior a 5 casos por cada 10.000 personas en la Comunidad Europea".

Los medios de comunicación contribuyen a crear una determinada imagen de los pacientes en la sociedad. Las asociaciones y los pacientes pueden lograr a través de ellos empatizar con los ciudadanos e influir en las decisiones gubernamentales. La teoría del framing o encuadre puede resultar la más adecuada para analizar la posible influencia de los pacientes en la opinión pública. Según Entman (1993: 52), "consiste en 
seleccionar algunos aspectos de la realidad percibida y hacerlos más salientes en el texto comunicativo". A través del análisis de los contenidos comprobamos mediante esta teoría que los medios de comunicación no sólo fijan la agenda pública, sino que también dictan implícitamente una forma de pensar sobre ciertos asuntos (Igartua y Humanes, 2004). McCombs y Shaw (1993) introducen un nuevo aspecto en el que se afirma que la forma en que nos cuentan las cosas sí que influye en la forma de percibir la realidad.

Esta investigación no parte del vacío, ya que otros autores y autoras ya han dedicado sus esfuerzos a analizar las temáticas de enfermedades raras y su cobertura a través de la prensa. En este sentido hay que destacar las investigaciones que aplican la teoría del framing como las de Sánchez Castillo (2012, 2013), que pone de manifiesto que la prensa, a través de sus textos y fotografías, muestra como representativo de las enfermedades raras a un grupo de personas desconsoladas, que buscan visibilidad y recursos económicos para investigación y demandas específicas. Sánchez Castillo (2013: 75) apunta a la necesidad de "una mejor canalización entre las instituciones sanitarias responsables y los profesionales de la información". Además, Sánchez Castillo y Mercado (2014: 123) afirman en un estudio posterior sobre los encuadres en prensa de las enfermedades raras que se representan "como una realidad social dentro de un contexto problematizado y no únicamente como un asunto médico".

Por otra parte, Urrutia (2013) realiza un análisis cuantitativo y cualitativo del Día Mundial de las enfermedades raras en Andalucía, en el que se aprecia la debilidad de las asociaciones de pacientes, que apenas figuran como voces reivindicativas y en la necesidad de que los medios de comunicación no recurran al morbo y pesimismo, característico en este tipo de información.

También es necesario reseñar otros estudios, como por ejemplo el de Bañón et al. (2011) sobre el tratamiento que los medios de comunicación ofrecen sobre las enfermedades raras elaborado por el CIBERER (Centro de Investigación Biomédica en Red de las Enfermedades Raras), los grupos de investigación ECCO (Universidad de Almería) y GIDYC (Universidad de CEU Cardenal Herrera). Se trata de una de los trabajos más exhaustivos, análisis cuantitativo-cualitativo de la prensa escrita impresa y digital española durante 2009 y 2010. Entre los aspectos a destacar de esta investigación se encuentran las propuestas de actuación para mejorar la escasa importancia en prensa o la presencia de fuentes oficiales políticas como portavoces de estos pacientes. Desde el año 2013 el Observatorio de Enfermedades Raras, OBSER, está vinculado al grupo de investigación Estudios Críticos de la Comunicación (CySOC de la Universidad de Almería), que desde años anteriores realiza un minucioso análisis sobre la comunicación verbal relacionada con estas patologías.

Bañón (2007: 222) otorga gran importancia al papel que los mass media juegan en la representación social de estas patologías. Los pacientes con ER son "considerados como un colectivo minoritario con escasa capacidad, por lo normal, para ejercer una influencia importante en los asuntos políticos o sociales. Por lo que han sido sistemáticamente excluidos de los foros de discusión más importantes".

Para Castillo et al. (2015: 689) la conexión con la sociedad de los pacientes con enfermedades raras sería más fácil a través de los medios, ya que "los periodistas tendrían mayor facilidad para obtener información directa de los propios afectados si se tuviera en cuenta la comunicación con estos profesionales".

Villa et al. (2014: 2), consideran que la presencia en los medios es "ecoica"; es decir, de repetición de informaciones de agencias y menos de profundización de la situación real de las enfermedades poco frecuentes. Pero sobre todo, interesa recoger en estas investigaciones cómo se informa, los buenos y malos modelos comunicativos sobre estas enfermedades minoritarias, con el fin de corregirlas y llegar a los 
profesionales de la información para que la imagen que aparece en los medios de comunicación sea lo más real y positiva para este colectivo.

Sin embargo, en la literatura señalada no es tan frecuente encontrar análisis que abarquen los encuadres que generan tanto los medios impresos como audiovisuales en España. En este contexto es donde esta investigación pretende contribuir con un acercamiento más genérico pero no menos exhaustivo al conocimiento de las prácticas comunicativas que diariamente se ofrecen en los distintos medios de comunicación en España sobre estas patologías.

\section{Objetivos}

Los principales objetivos de esta investigación se basan en:

1. Analizar los encuadres o frames sobre las enfermedades raras que predominan en prensa, radio y televisión en el periodo de 2012 a 2014 en los medios con mayor difusión o audiencia en España.

2. Comparar estos encuadres en los en los distintos medios analizados.

3. Analizar si existe variación en el tratamiento de estos encuadres teniendo como referencia 2013 como el Año Español de las Enfermedades Raras.

\section{Metodología}

Muestra

Nos basamos para obtener los resultados de esta fase en el análisis de contenido de 527 piezas sobre enfermedades raras, se trata de las unidades localizadas en estos medios tras filtrar su relación con el objetivo de nuestro análisis en los distintos medios: prensa, radio y televisión, de 2012 a 2014.

Los medios seleccionados responden a los criterios de publicaciones de mayor difusión en prensa, según la Oficina de Justificación de la Difusión en España, OJD. Analizamos las piezas que aparecen en El País, EI Mundo, ABC, La Vanguardia y El Periódico.

Tabla 1: Difusión de los diarios españoles 2012 a 2014

Fuente: $O J D$

\begin{tabular}{|l|c|}
\hline Diario & Promedio de Difusión \\
\hline El País & 276.883 \\
\hline El Mundo & 156.172 \\
\hline La Vanguardia & 145.873 \\
\hline ABC & 134.553 \\
\hline El Periódico & 96.162 \\
\hline
\end{tabular}


El periodo analizado abarca los años 2012, 2013 y 2014, de enero a diciembre. La selección de los contenidos se ha hecho a través de las herramientas especializadas de búsqueda hemerográfica MyNews, Kiosko y Más y Orbyt. ${ }^{1}$

En un primer rastreo se obtuvieron 1161 informaciones de temática relacionada con enfermedades raras, que quedaron reducidas a 276 tras una segunda lectura en la que se extrajeron únicamente aquellos textos basados en noticias y reportajes relacionados con el colectivo de pacientes que padecen estas enfermedades minoritarias

En el caso de las emisoras de radio y televisión seleccionamos las que tienen mayor audiencia según el EGM, Estudio General de Medios: Cadena Ser, Onda Cero, Cope y RNE, en radio. Y en televisión: Telecinco, Antena 3, TVE, La Sexta, Cuatro.

Para analizar las piezas de radio se seleccionaron los podcasts sobre enfermedades raras a través de las páginas web de los medios que forman parte de la muestra en el periodo de 2012 a 2014 . Se obtuvo como resultado 157 piezas para analizar.

Tabla 2: Audiencia de las emisoras de radio de 2012 a 2014

\begin{tabular}{|l|c|}
\hline Emisoras & Oyentes \\
\hline Cadena Ser & 4.725 .000 \\
\hline Onda Cero & 2.632 .000 \\
\hline Cope & 1.746 .000 \\
\hline RNE & 1.267 .000 \\
\hline
\end{tabular}

Fuente: EGM2014

El criterio para realizar la búsqueda en televisión se realizó a través de las páginas web de estos medios que forman parte de la muestra. Se realizó la búsqueda de todas aquellas piezas sobre enfermedades raras en las páginas y se comprobó, de las 150 que se encontraron, que estuvieran relacionadas con las patologías y pacientes, aunque finalmente se realizó un visionado para comprobar el contenido de las mismas y quedó reducido a 94 piezas televisivas.

Tabla 3: Audiencia de las emisoras de televisión de 2012 a 2014

\begin{tabular}{|l|c|}
\hline Emisora de televisión & $\begin{array}{c}\text { Promedio Audiencia/ } \\
\text { Share }\end{array}$ \\
\hline Telecinco & $18,4 \%$ \\
\hline Antena 3 & $16,9 \%$ \\
\hline La 1 & $13,4 \%$ \\
\hline La Sexta & $7,2 \%$ \\
\hline Cuatro & $6,5 \%$ \\
\hline
\end{tabular}

Fuente: EGM2014

\footnotetext{
${ }_{1}^{1}$ My News es un recurso electrónico de información al que se puede acceder a través de las bibliotecas públicas y que presenta los textos completos de las noticias publicadas en la prensa española. Kiosko y Más es una editorial online que mediante suscripción permite acceder a periódicos y revistas españolas. Orbyt es el quiosco digital de Unidad Editorial, la editorial del diario El Mundo, que es objeto de análisis y a la que se accede mediante suscripción.
} 
Los años analizados tanto en radio como en televisión han sido los mismos que en prensa, 2012, 2013 y 2014, tomando como criterio un año anterior y otro posterior a la celebración del Año Español de las Enfermedades Raras. Una vez seleccionado el corpus de la investigación se ha recurrido al análisis de contenido, una técnica considerada fundamentalmente cuantitativa, aunque tiene un aspecto cualitativo tras el resultado de su aplicación. Se analizan todas las noticias sobre enfermedades raras que surgen en el que se considera oficialmente Año Español, desde el 1 de enero al 31 de diciembre de 2013 y en el año anterior, 2012 y el posterior, 2014, también los doce meses. Se tiene en cuenta que en 2014 se producen algunos actos relacionados con las iniciativas de 2013. Y durante 2014 se pretende comprobar si ha variado 0 aumentado la repercusión de estos actos organizados.

\section{Análisis de contenido}

Realizamos un análisis de contenido de las piezas en prensa, radio y televisión. El análisis de contenido es: "un conjunto de procedimientos interpretativos y de técnicas de comprobación y verificación de hipótesis aplicados a productos comunicativos (mensajes, textos o discursos) o a interacciones comunicativas que, previamente registradas, constituyen un documento, con el objeto de extraer y procesar datos relevantes sobre las condiciones mismas en que se han producido, o sobre las condiciones que puedan darse para su empleo posterior" (Piñuel, 1999:519).

Nos basamos en la clasificación de frames procedentes de diversos estudios (Semetko y Valkenburg, 2000, Rodero et al., 2009):

a) Frame de responsabilidad. Se registran todas las manifestaciones que atribuyan la responsabilidad de la situación de los pacientes con enfermedades raras.

b) Frame de interés humano. Se engloban las historias personales de los pacientes y sus familiares.

c) Frame de movilización. Implica movilización por parte de las organizaciones de enfermedades raras.

d) Frame de consecuencias económicas. Informaciones relativas a las repercusiones económicas, pérdidas o ganancias.

f) Frame basado en la clasificación de Liu y Kim (2011), frame científico; en el que tendremos en cuenta a su vez los encuadres de detección de la enfermedad, prevención de la enfermedad, factores de riesgo en el estilo de vida y descubrimiento científico.

En el caso de los frames visuales establecemos 7 encuadres visuales: VF1 "imagen de pacientes de forma individual", VF2 "imagen de personalidades que apoyan a los pacientes", VF3 "investigadores, genetistas o médicos", VF4 "imagen de los representantes de las asociaciones de ER con afectados", VF5 "imagen de políticos con afectados", VF6 "miembros de instituciones relacionadas con ER" y VF 7 "imagen de familiares con pacientes". 


\section{Libro de Códigos}

Tras la selección de un sistema de categorías, se elaboró un libro de códigos (codebook), bajo criterios de claridad y precisión, teniendo en cuenta una ficha de análisis con las siguientes variables:

1. Datos identificativos básicos; identificación del periódico, radio y emisora de televisión en que aparece la pieza, mes de difusión, localización de la información, sección (ya sea en prensa, en la programación de radio y televisión) y espacio que ocupa (en prensa la longitud de la información y en radio y televisión la duración de las unidades de análisis).

2. Indicadores relacionados con el contenido y tema (issue). Se codifica la temática en grupos; relacionados con interés humano, si está relacionada con actos organizados sobre estas enfermedades, económico (desde el punto de vista de la situación económica de los afectados), científico (con relevancia para la divulgación o tratamiento de la enfermedad), político y otros temas. A partir de esta temática, encuadramos estas piezas en frames de interés humano, económico, científico, movilización social, responsabilidad política, conflicto y moralidad. Con respecto al contenido analizamos el género (informativo, reportaje, crónica, entrevista, opinión).

3. Fuentes que genera la información: políticos, científicos, médicos, instituciones relacionadas con las enfermedades raras, miembros de las asociaciones, Feder, farmacéuticas, afectados a título individual, personalidades, otros.

4. Imágenes: tipología; fotografía, tablas, gráficos y temática de las mismas (en prensa). En televisión, tipo de planos utilizados (nos basamos en si el montaje es analítico: planos cortos y de breve duración, expresivos y psicológicos y sintético: planos más largos y profundidad de campo que muestran una visión más completa de la realidad o la mezcla de ambos). Ámbito donde se desarrolla la acción (público, privado o ambos).

\section{Resultados}

Cobertura en prensa, radio y televisión

Los primeros datos que obtenemos mediante el análisis en prensa están relacionados con la cobertura en los distintos diarios analizados. El medio que más noticias genera sobre ER es la prensa con un $57,32 \%$, seguido de la radio $(29,79 \%)$ y la televisión $(17,83 \%)$. Esto significa que las organizaciones tienen más facilidad en conseguir presencia escrita, lo que hace que deban trabajar más en la elaboración de informaciones radiofónicas y televisivas, puesto que por estos medios disponen de sonido (en el caso de las emisoras de radio) o de sonido e imagen (en las televisiones), lo que supone mayores posibilidades de presencia mediática.

En prensa, el diario que mayor cobertura da a las enfermedades raras es el diario $A B C$, con el $44 \%$ de la información durante el periodo de análisis y el que menos cobertura ofrece es el diario El Periódico, genera un $9 \%$ de las piezas.

En radio, la cadena pública, $R N E$, genera el $42 \%$ de las piezas analizadas, es la emisora que presta mayor atención a estas patologías, mientras que le presta menor atención es Onda Cero. Y en televisión se produce el mismo fenómeno, es la cadena pública la que mayor compromiso demuestra con el colectivo de pacientes 
y estas enfermedades. Esto se debe al acuerdo que el ente público RTVE tiene en el año 2012, ya que el Ministerio de Sanidad de España y estas organizaciones llegan a un acuerdo para emitir una serie de programas de radio y televisión de divulgación sobre estas patologías con objeto de favorecer su repercusión social. Cerca de la mitad de las piezas las produce La Primera de TVE, y el 16\% La 2 de TVE. Por tanto, el $56 \%$ de los espacios proceden de $T V E$, frente a $L a$ Sexta con el $5 \%$.

Gráfico 1: Cobertura en prensa, radio y televisión

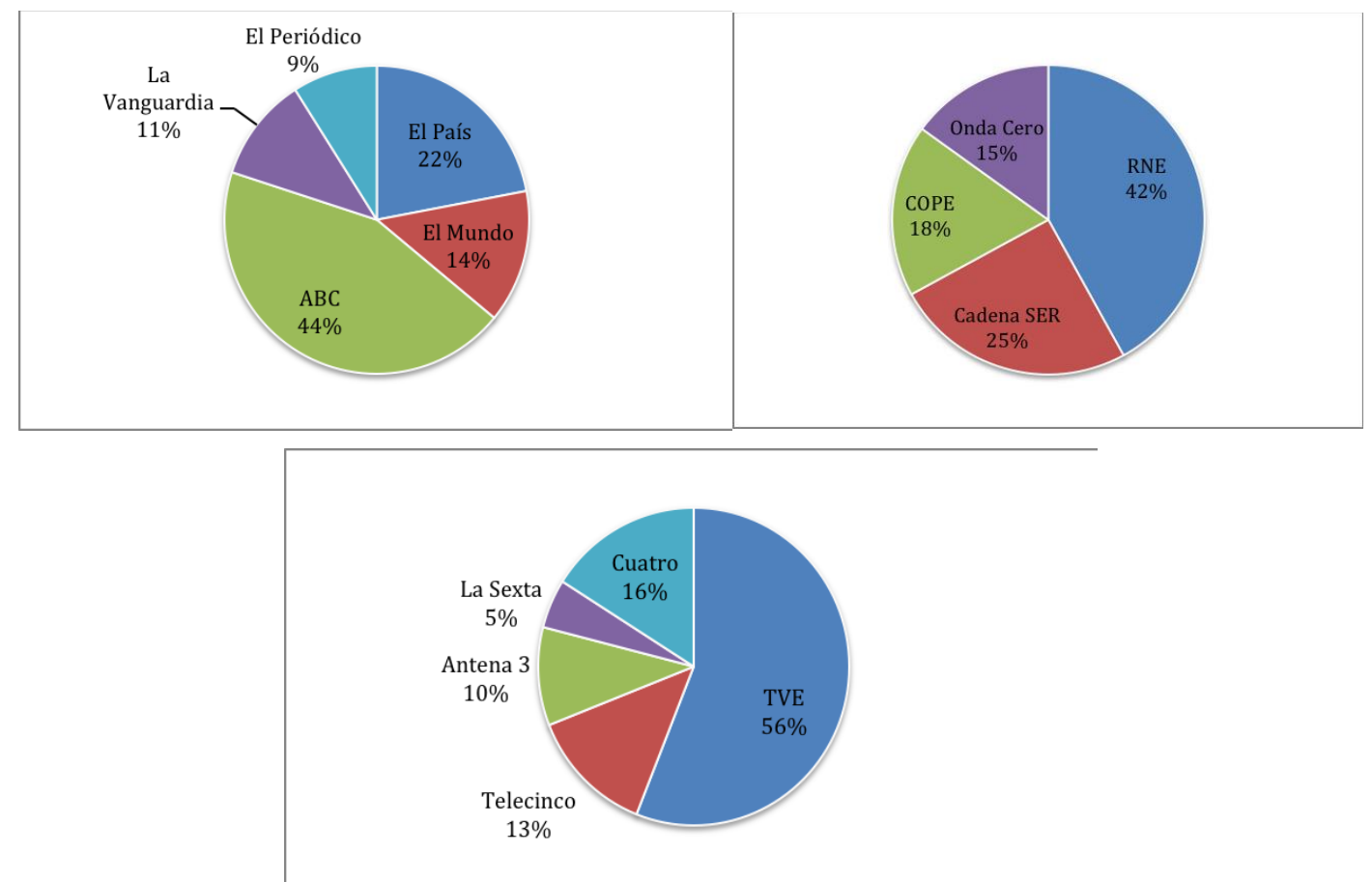

Fuente: elaboración propia

En prensa, coincidiendo con el Año Español de las Enfermedades Raras, se cubren mayor número de noticias sobre estas patologías en 2013. Hay un incremento notable de la cobertura con respecto a 2012 y en 2014 el número de noticias es menor que en 2013.

En radio hay mayor cobertura en 2014, el 50\%, seguido de 2013 y 2012. En televisión, el año 2013 marca un cambio de tendencia con respecto al año 2012, un aumento en la cobertura sobre enfermedades raras, que se consolida en 2014. 
Gráfico 2: Cobertura por años en prensa, radio y televisión

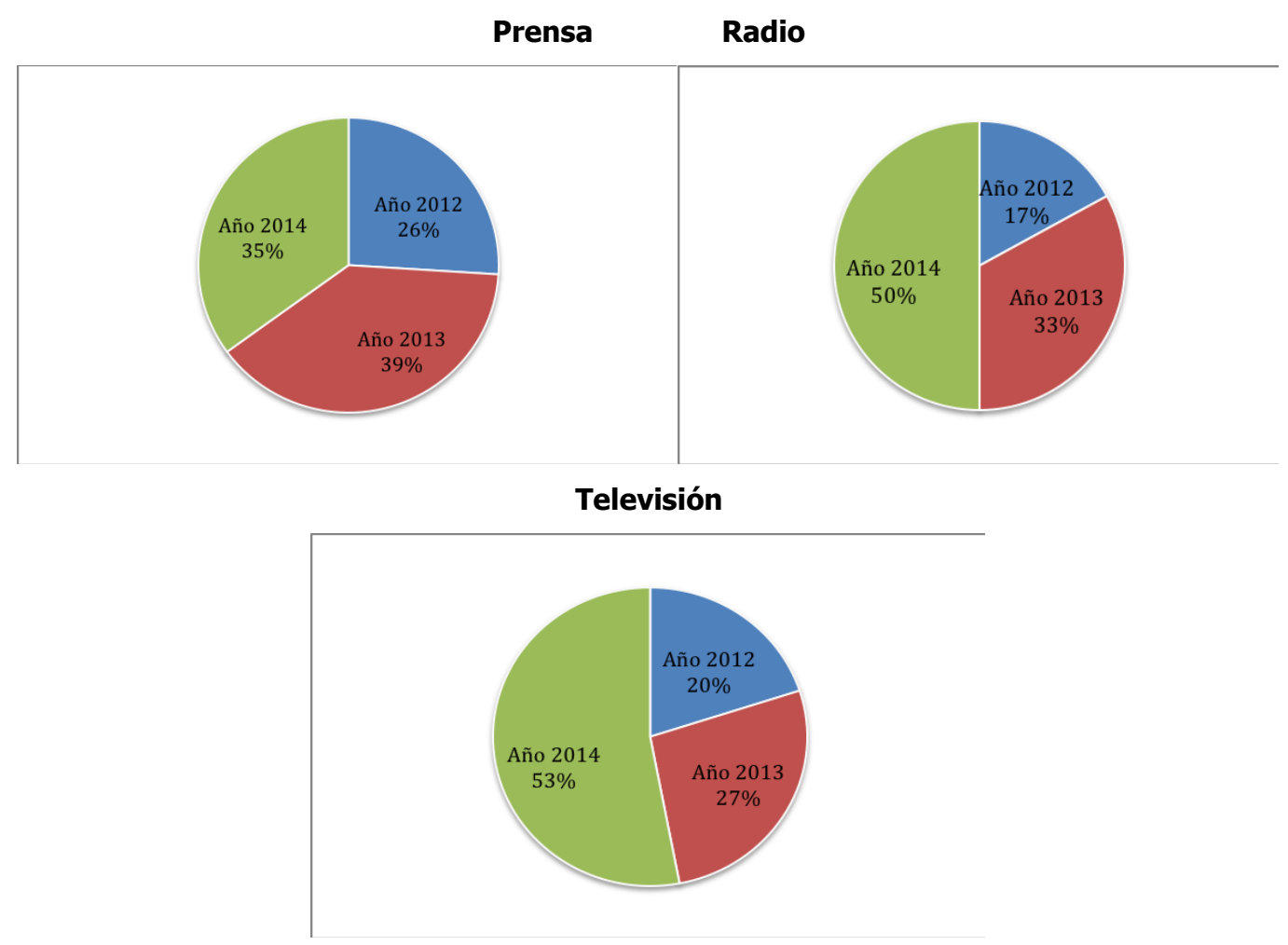

Fuente: elaboración propia

El mes de febrero es tradicionalmente el mes que más porcentajes de espacios se emiten tanto en prensa, radio como en televisión. Esto se debe a la celebración del Día Mundial de las Enfermedades Raras, que en 2012 tiene lugar el 29 de febrero y en 2013 y 2014, el día 28 de febrero. La tendencia por meses tanto en radio y televisión es que hay un mayor porcentaje de programas e informativos el mes anterior y posterior a febrero, en enero y marzo, y en diciembre, un mes en el que habitualmente se realizan actos solidarios para recaudar fondos.

\section{Ubicación de las piezas y duración}

En el año 2012, el 70\% de las unidades de análisis se sitúa en página par y el 30\% en impar. En 2013 las piezas aparecen el $52 \%$ en páginas pares y el $48 \%$ en impares. En el año 2014 , el $60 \%$ de las noticias está en página par y el $40 \%$ en página impar. Se le concede mayor importancia a las páginas impares en prensa, ya que las páginas del periódico se abren de derecha a izquierda, lo que contribuye a que el primer golpe de vista recaiga sobre la página izquierda, que es donde se suelen ubicar las noticias más importantes y la publicidad es más cara en este emplazamiento.

Determinamos los encuadres textuales estableciendo las unidades de análisis en las secciones y espacios que están destinadas a contener esta información. En prensa, la sección predominante es Sociedad en los años 2012 y 2013, donde se ubican el 36\% y el 24\% de las piezas. Sin embargo, en 2014 hay un cambio 
de tendencia, la sección con más noticias es una sección Especial en Ciencia, donde se ubica el 21\% de estas informaciones mientras que en Sociedad sólo aparece el 8\%. En el género Opinión también se trata la temática de las enfermedades raras, y es en 2013 donde más piezas de Opinión se producen, el 13\%. De ello podemos deducir que no es hasta 2014 cuando se categorizan esas piezas dentro de un espacio sanitario o científico, puesto que en 2012 y 2013 aparecían encuadradas en una sección como Sociedad, considerada en el gran 'cajón de sastre' donde aparecen noticias de diversa índole sin una clasificación específica.

La mayoría de las piezas ocupan poco espacio, la media es menos de un cuarto de página en los tres años, 2012, 2013 y 2014. Por otra parte, en 2012 hay un $42 \%$ de piezas que ocupan media página y tres cuartos. En el año 2013 ocupa menos de un cuarto de página el 34\% y en 2014, el 45\%. Va disminuyendo el espacio que ocupan estas informaciones conforme avanzan los años. Hay un mayor número de noticias, pero se les concede menos espacio.

Si atendemos al porcentaje de portadas en prensa, sólo hay un $1 \%$ en 2013, y se produce con el tratamiento del juicio por el caso de la talidomida. Ni siquiera se trata esta noticia desde el punto de vista de que es una enfermedad rara. La expectación mediática se produce porque más de 50 años después los afectados solicitan a la empresa farmacéutica alemana una cantidad indemnizatoria por los daños producidos. El tratamiento que se le otorga a estas noticias está relacionado con una cuestión de justicia o negligencia farmacéutica y la demanda millonaria de sus afectados. Por tanto, no hay referencia en portadas a los actos relacionados con el Año Español de las Enfermedades Raras. En los años 2012 y 2014 ninguna de las noticias sobre Enfermedades Raras se refleja en portada, por lo menos no refiriéndose a las patologías como tal, aunque sí que una portada en 2014 sobre el reto del cubo helado debido a un suceso, no por la patología. Existe además, durante 2014, una entrevista en la contraportada relacionada con el caso de una paciente que padece un síndrome de parálisis facial denominado Moebius.

La duración de los espacios en radio de forma mayoritaria oscila entre los 5 y 30 minutos. Eso es lo que duran el 57\% de las piezas analizadas, también duran entre 1 y 5 minutos el 27\%, menos de 1 minuto, el $14 \%$ y más de 30 minutos el $2 \%$. En radio se le dedica mayor espacio a los contenidos sobre enfermedades raras, puesto que más de la mitad de las piezas duran más de cinco minutos. Los ubicamos en programas el $76 \%$ y en informativos el $24 \%$ de las unidades de análisis. El $68 \%$ tiene carácter nacional, el $19 \%$ son espacios locales, el $11 \%$ se emiten en emisoras regionales y el $2 \%$ en sección internacional (espacios de Radio Exterior de España, REE). Por tanto, los contenidos sobre enfermedades raras se tratan en programas, entrevistas, en los que se concede mayor profundidad a la temática y son más cercanos a la audiencia, puesto que cerca del $70 \%$ se transmite en ámbito regional.

La duración de estos espacios en televisión tiene una media de entre 1 y 5 minutos, ( $44 \%$ de las piezas), hay un $28 \%$ que duran entre 5 y 30 minutos, el $22 \%$ dura menos de 1 minuto y un $6 \%$ dura más de 30 minutos.

En televisión, el $49 \%$ son piezas de informativos, el $48 \%$ son programas y el $3 \%$ son espacios publicitarios o campañas sobre enfermedades raras. La televisión trata la temática en espacios breves, habitualmente en las noticias de los informativos o como un espacio breve en un programa. El 84\% pertenecen al ámbito nacional, (regionales el $16 \%$ restante). Por tanto, son espacios que se han dirigido mayoritariamente a toda la población en programas e informativos nacionales de la cadena con una gran repercusión mediática. 


\section{Géneros y contenidos}

El género que más se utiliza en prensa es la noticia (72\%). El resto de géneros utilizados en prensa son: Opinión (13\%), reportaje (12\%) y entrevista (3\%). El género predominante en radio es la entrevista, el $50 \%$ de los espacios. El $22 \%$ son noticias, el $14 \%$ son reportajes, el $8 \%$ son tertulias, el $3 \%$ crónicas, el $2 \%$ son debates y el $1 \%$ son informes. El tipo de mensaje es de carácter informativo, $52 \%$ frente a mensajes testimoniales, $26 \%$ y persuasivos, $22 \%$. Por otra parte, en televisión, el género predominante es la noticia, $35 \%$, el $32 \%$ son reportajes, el $26 \%$ son entrevistas, el $6 \%$ son publirreportajes y $1 \%$ son documentales. En el $50 \%$ de las piezas televisivas predomina un mensaje testimonial, el $40 \%$ de los mensajes son persuasivos y un $10 \%$ tiene carácter predominantemente informativo. Se trata de noticias que aparecen en los informativos sobre estas patologías y también hay un tanto por ciento importante de reportajes que aparecen en programas e informativos. Por tanto, prensa y televisión tratan las enfermedades raras como noticias o reportajes, en el caso de la televisión frente a la radio que da cabida a la temática en las entrevistas.

Al realizar una primera clasificación, a rasgos generales, ordenamos las temáticas de las noticias en diversos grupos entre los que se encuentran; contenido de interés humano, económico, científico, político, actos organizados y otros. Algunas tienen en común varias temáticas, aunque hemos resaltado las principales en las distintas unidades de análisis. En prensa, las noticias de interés humano son las más frecuentes, $42 \%$, seguidas de las noticias de actos organizados, $28 \%$ y las noticias científicas, $24 \%$. Las noticias sobre temas económicos también son relevantes en el periodo de análisis, $23 \%$ y por último el $8 \%$ son de carácter político y sobre otros temas el $1 \%$. El frame de interés humano se impone en prensa frente a otros encuadres, le sigue el frame de movilización con la temática de actos organizados, en tercer lugar encontramos el encuadre científico y en cuarto lugar, el encuadre económico. Las noticias que tienen un carácter político, en las que se habla sobre responsabilidad de una determinada situación tienen un menor porcentaje que el resto.

Por regla general, se enfatizan una realidad social y, aunque se difunde también información científica, pesa más como realidad social en una situación problemática agravada por la crisis económica. El interés humano prevalece con titulares impactantes como "Le quedan cinco años de vida y no puedo darle todo lo mejor", (13 de febrero de 2013 en ABC), "La enfermedad de mi hija nunca iba a ser investigada", (4 de junio de 2013 en El País), "Que me acompañen y no se rían de mí", (3 de diciembre de 2013 en ABC). 
Imagen 1: Reportaje social y económico del diario El País

\section{Una cuestión de vida o muerte para las niñas Paula y Marta}

El Gobierno ha impuesto el copago en los dietoterápicos e Muchos enfermos necesitan estos caros productos para sobrevivir e Algunos no podrán pagarlos
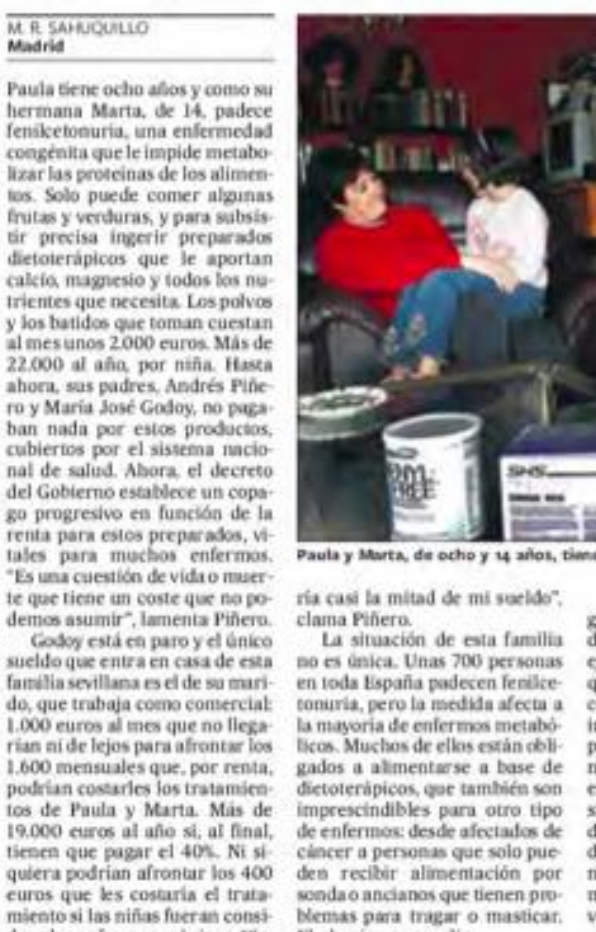

Fuente: El País, 29 de abril de 2012

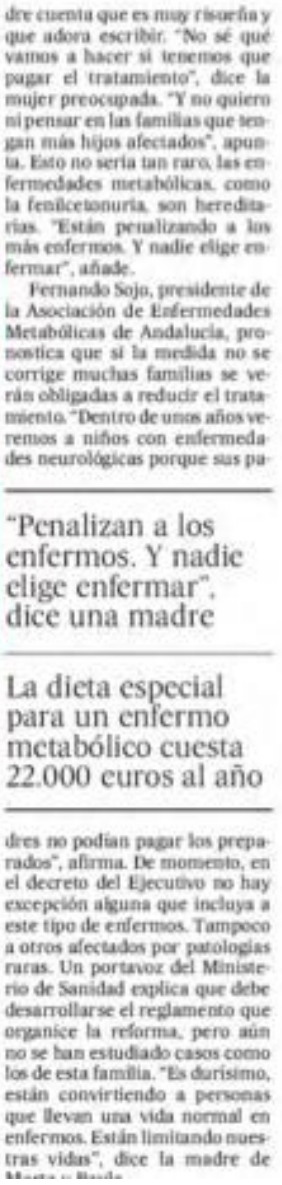

dre turnta que es migy risur hiay paie adona escribir: No se quit pagar el tratamiento:", dice la mujer preocupada. Yno quiemi kan más hijas afectados., apue Cester it fenilcetopurts pos beredia in. Testin prializando a law mis esfermon $Y$ nadie clise es Fernando Soja, incsidente de Metibelicas de Andeluels gre mostica gue si la medida no se

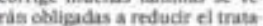

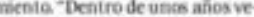
des neuroldagicas porque sus pa

"Penalizan a los enfermos. Y nadic elige enfermar dice una madre

La dieta especial para un enfermo metabólico cuesta 22.000 euros al año

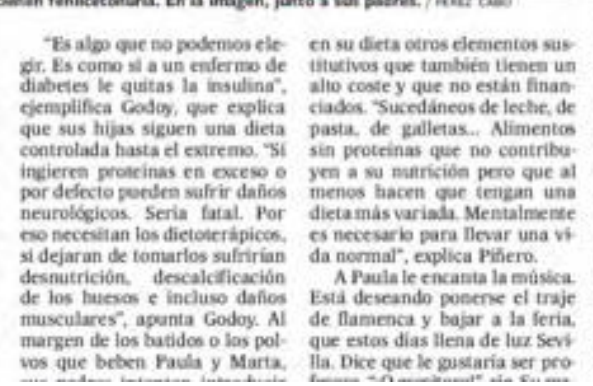

dres no podtan pagar las prepa. excepcibn atguna que incluya a te enfermos Tampocois del Ministe. desamottar se el replamenafo que stin convirtiendo a persenas gue llevan una vida normal et ras vides", dece la modre de thas videsis dice

Sin embargo, también se producen otros titulares que tienen que ver con la movilización con los que se rompe ese imaginario de pacientes desvalidos son: "Somos raros, ¿̇y qué?", (24 de febrero de 2014 en ABC), "Los afectados por dolencias raras toman la iniciativa", (28 de febrero de 2014 en El País), "Pocos, pero no solos", (4 de marzo de 2014 en La Vanguardia) o "Imaginación para financiar la ciencia en crisis", (23 de marzo de 2014 en El Mundo). 
Imagen 2: Reportaje "Somos raros, ¿̇y qué?"

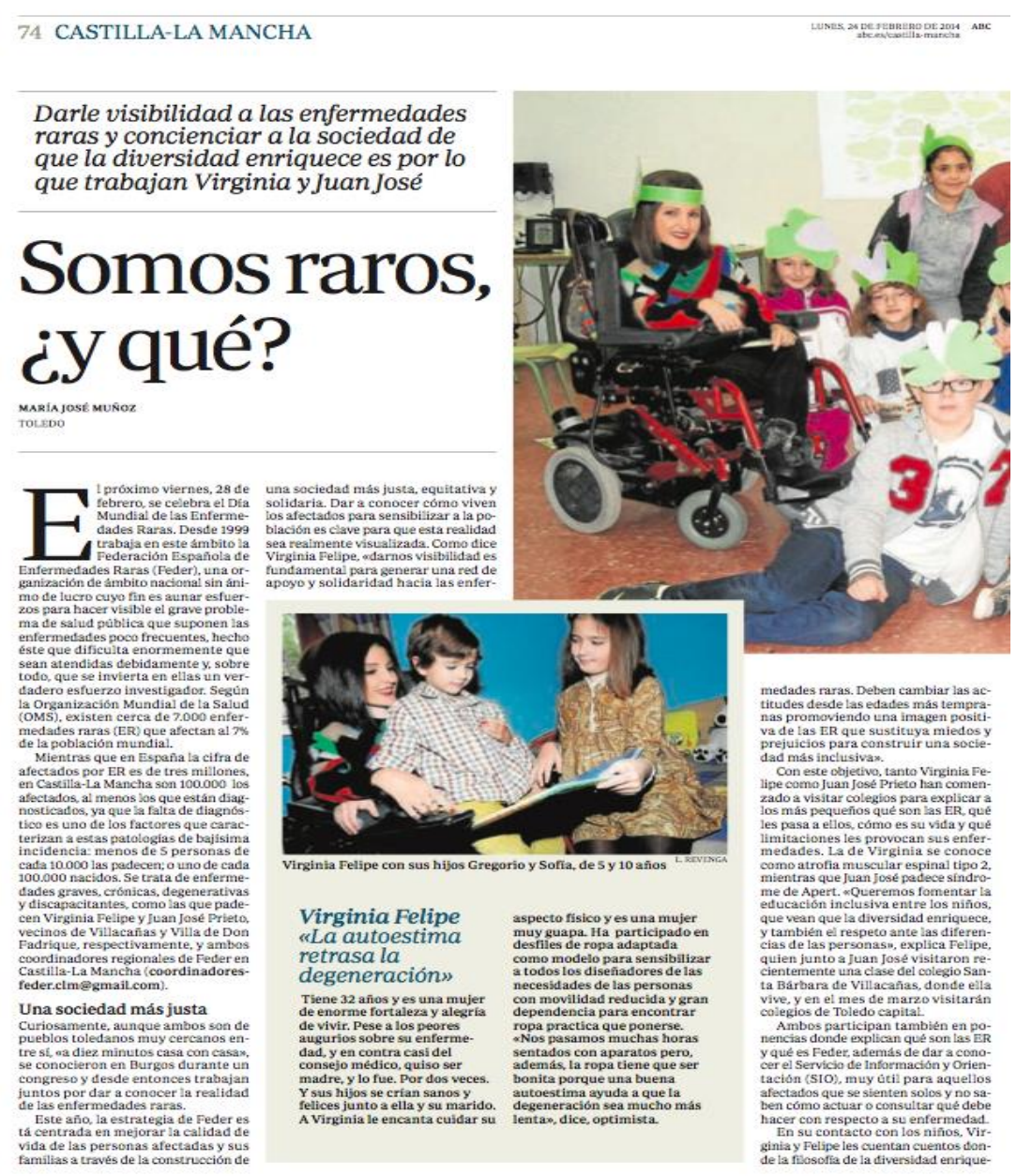

Fuente: $A B C, 24$ de febrero de 2014

Los temas que más se tratan en radio son actos organizados, 50\%, bien con motivo del Año Español de las Enfermedades Raras, del Día Mundial de estas patologías o de actos para recaudar fondos. Le siguen los espacios de interés humano, 46\%, (suelen ser entrevistas a personas que padecen la enfermedad). Se trata la divulgación científica, 24\%, (en espacios de salud que generalmente ofrece $R N E$, la radio pública, aunque también existen programas de salud en otras emisoras privadas pero en menor medida), los recortes y los problemas económicos tienen lugar en el $20 \%$ de las piezas analizadas, desde un prisma político se analizan en el $5 \%$ y un $2 \%$ trata las enfermedades raras desde otro enfoque.

En televisión, la temática de interés humano, 63\%, está por encima de los actos organizados, 53\%. El interés humano es propio de espacios en los que se trata historias personales de enfermos o familiares, situaciones sociales y económicas y aspectos reivindicativos basados en la atención sociosanitaria. Los temas económicos se tratan en el $21 \%$ de los espacios y normalmente están relacionados con los recortes que padece el colectivo. La temática científica o frame científico del $18 \%$ de los espacios se caracteriza por explicar la patología, su sintomatología y diagnóstico o terapias innovadoras basada en investigaciones científicas, en el mejor de los casos. La política o frame de responsabilidad tiene poco que ver con estas enfermedades, puesto que los espacios que tratan las patologías desde el punto de vista político son escasos 
y el interés se suele centrar en los pacientes o sus reivindicaciones mediante actos que realizan para conseguir sus fines

Tabla 3: Frames predominantes en prensa radio y televisión

\begin{tabular}{|l|c|c|c|}
\hline Temáticas & Prensa & Radio & Televisión \\
\hline Movilización & $28 \%$ & $50 \%$ & $53 \%$ \\
\hline Científico & $24 \%$ & $24 \%$ & $18 \%$ \\
\hline Responsabilidad-(Política) & $8 \%$ & $5 \%$ & $2 \%$ \\
\hline Consecuencias Económicas & $23 \%$ & $20 \%$ & $21 \%$ \\
\hline Interés Humano & $41 \%$ & $46 \%$ & $63 \%$ \\
\hline
\end{tabular}

Fuente: Elaboración propia

\section{Fuentes de información}

Las fuentes de información en prensa son fundamentalmente las organizaciones de pacientes (asociaciones), $43 \%$ y los médicos e investigadores, 32\%. Hay otras fuentes sin determinar, entre las que destacamos a partir del Año Español de las Enfermedades Raras, las fuentes de la Casa Real o el ente público RTVE, que informa sobre los actos organizados en sus emisoras de radio y televisión. El resto de fuentes a las que se recurren son: los políticos, 17\%, pacientes a título individual, 14\%, las farmacéuticas, 14\% y otras personalidades, $2 \%$.

Con respecto a los personajes que aparecen en radio, predominan los que actúan a título individual, en el $66 \%$ de los espacios y el $54 \%$ representan a un colectivo. Los roles o personajes más frecuentes en radio son los miembros de asociaciones, 50\%, seguido de médicos e investigadores, $30 \%$. Los familiares, $26 \%$, y pacientes, $21 \%$, también ocupan un lugar destacado en la radio. Son fuentes de las que se nutren en los programas radiofónicos para contar a través de testimonios las circunstancias de los pacientes. Las personalidades aparecen como personajes importantes que apoyan la temática sobre la que gira el tema central de las diversas entrevistas radiofónicas, por ejemplo, la periodista Isabel Gemio, el humorista Pedro Ruiz, el cantante Bertín Osborne, son personalidades que apoyan la causa y que la hacen visible a los oyentes. La presencia de los políticos es menor y normalmente en estos espacios están acompañados por miembros de organizaciones para tratar temas concretos como la celebración de un acto, la participación de una administración en una iniciativa que les afecte, etc.

En el $88 \%$ de las piezas hay personajes a título individual pero también hay un $44 \%$ de piezas en la que los protagonistas son personajes que representan a un colectivo. Por tanto, aparecen de forma individual y representando a colectivos de forma conjunta en ambos medios, aunque se da más importancia a las personas que a las asociaciones. Los protagonistas son los pacientes como sujetos de interés humano, 62\%, y los familiares, $59 \%$.

En el caso del medio televisivo los pacientes a título individual aparecen en más espacios que los miembros de las asociaciones, 44\%, a diferencia de la radio. Las personalidades también aparecen más en la televisión, $38 \%$, que en otros medios. Siempre es más llamativo para el medio que el acto que se cubre cuente con la presencia de una figura pública, como la reina Doña Letizia, personajes del mundo de la cultura como la 
escritora Almudena Grandes, los cantantes Ana Belén, Joan Manuel Serrat, o el deportista, Rafa Nadal. Los políticos tienen menor presencia, $9 \%$, un porcentaje parecido al resto de medios.

\section{Los frames visuales}

En los encuadres visuales destacamos en prensa que el $94 \%$ son fotografías, el $5 \%$ son gráficos y otro tipo de imágenes el 1\%. Los frames visuales en prensa nos muestran en 2012 y 2013 a pacientes que reivindican una mejora y mayor visibilidad en la vida pública y en la agenda política. Y en 2014 se destaca más la presencia de personalidades y los científicos y médicos ejercen el papel de protagonistas para reivindicar mayor atención a estas enfermedades y a la búsqueda de financiación y atención sociopolítica para normalizar la situación de los pacientes. Por número de apariciones en las unidades de análisis, destacamos que los protagonistas de los encuadres en el 33\% de las imágenes son los pacientes que aparecen representados de forma individual, seguidos de personalidades que apoyan la causa, tal y como vemos en la tabla 4.

Tabla 4: Frames visuales en prensa de 2012 a 2014

\begin{tabular}{|l|c|}
\hline Frames Visuales & Porcentaje Total \\
\hline VF- 1. Pacientes & $33 \%$ \\
\hline VF- 2. Personalidades con afectados & $20 \%$ \\
\hline EV- 3. Investigadores/ genetistas o médicos & $14 \%$ \\
\hline EV- 4. Miembros de las asociaciones con \\
afectados & $8 \%$ \\
\hline EV- 5. Políticos con miembros de asociaciones & $5 \%$ \\
\hline EV- 6. Miembros de instituciones relacionadas & $2 \%$ \\
con ERs & $10 \%$ \\
\hline EV- 7- Familiares con pacientes & \\
\hline
\end{tabular}

Fuente: elaboración propia

La imagen de la reina Doña Letizia es una de las más frecuentes entre las figuras públicas, junto a la imagen de periodistas como Isabel Gemio, personalidades del mundo de la cultura y deporte, como Miguel Ríos o el ex seleccionador nacional, Vicente del Bosque. 
Imagen 3: Fotografía de la reina Doña Letizia en $A B C, 1$ de marzo de 2014

10 ENFOQUE

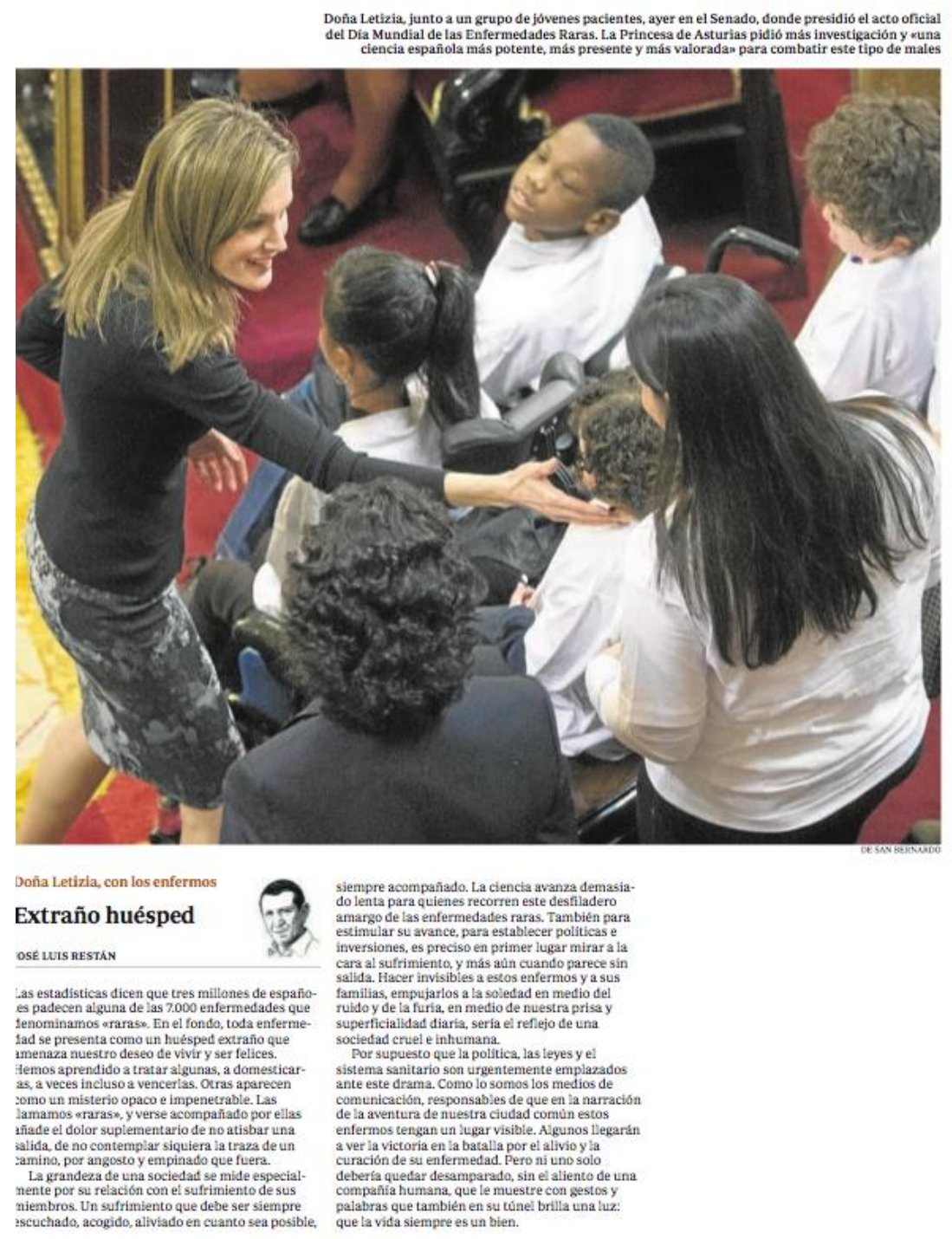

Fuente: $A B C, 1$ de marzo de 2014

En televisión los protagonistas son los pacientes como sujetos de interés humano, $62 \%$, y los familiares que les acompañan, 59\%. En el caso del medio televisivo los pacientes a título individual aparecen en más espacios que los miembros de las asociaciones, 44\%. Las personalidades también aparecen más en la televisión, 38\%, que en prensa. Siempre es más llamativo para el medio que el acto que se cubre cuente con la presencia de una figura pública, como la reina Doña Letizia, personajes del mundo de la cultura como Almudena Grandes, de la música, como Ana Belén y el cantautor Joan Manuel Serrat, o el deporte, Rafa Nadal. Los políticos tienen menor presencia, 9\%, un porcentaje ligeramente superior al de la prensa. Vemos, por tanto, la escasa implicación de los políticos en los asuntos relacionados con el colectivo de pacientes. 
Tabla 5: Frames visuales en televisión, periodo de 2012 a 2014

\begin{tabular}{|l|c|}
\hline Frames Visuales & Porcentaje total \\
\hline EV- 1. Pacientes & $62 \%$ \\
\hline EV- 2. Personalidades con afectados & $38 \%$ \\
\hline EV- 3. Investigadores/ genetistas o médicos & $26 \%$ \\
\hline EV- 4. Miembros de las asociaciones con afectados & $44 \%$ \\
\hline EV- 5. Políticos con miembros de asociaciones & $9 \%$ \\
\hline EV- 6. Miembros de instituciones relacionadas con & $44 \%$ \\
ERs & $59 \%$ \\
\hline EV 7- Familiares con afectados & \\
\hline
\end{tabular}

Fuente: elaboración propia

En lo que respecta a las características de las piezas en televisión destacamos que abundan las noticias y programas con un montaje analítico, es decir, se recurre a planos cortos de breve duración, expresivos y psicológicos en el $58 \%$ de las piezas. El $28 \%$ se basa en una edición y realización sintética, con planos más largos y profundidad de campo que muestran una visión más completa de la realidad. Y hay un $14 \%$ de unidades que mezclan ambos tipos de planos.

El ámbito público impera sobre el privado. El 77\% de los espacios analizados son programas, reportajes o noticias que se graban o emiten en escenarios o espacios públicos como centros sanitarios, escolares, lugares donde se celebran congresos, platós de televisión, etc.

Imagen 4: Programa Saber Vivir de TVE sobre el Día Mundial de las Enfermedades Raras en el año 2012

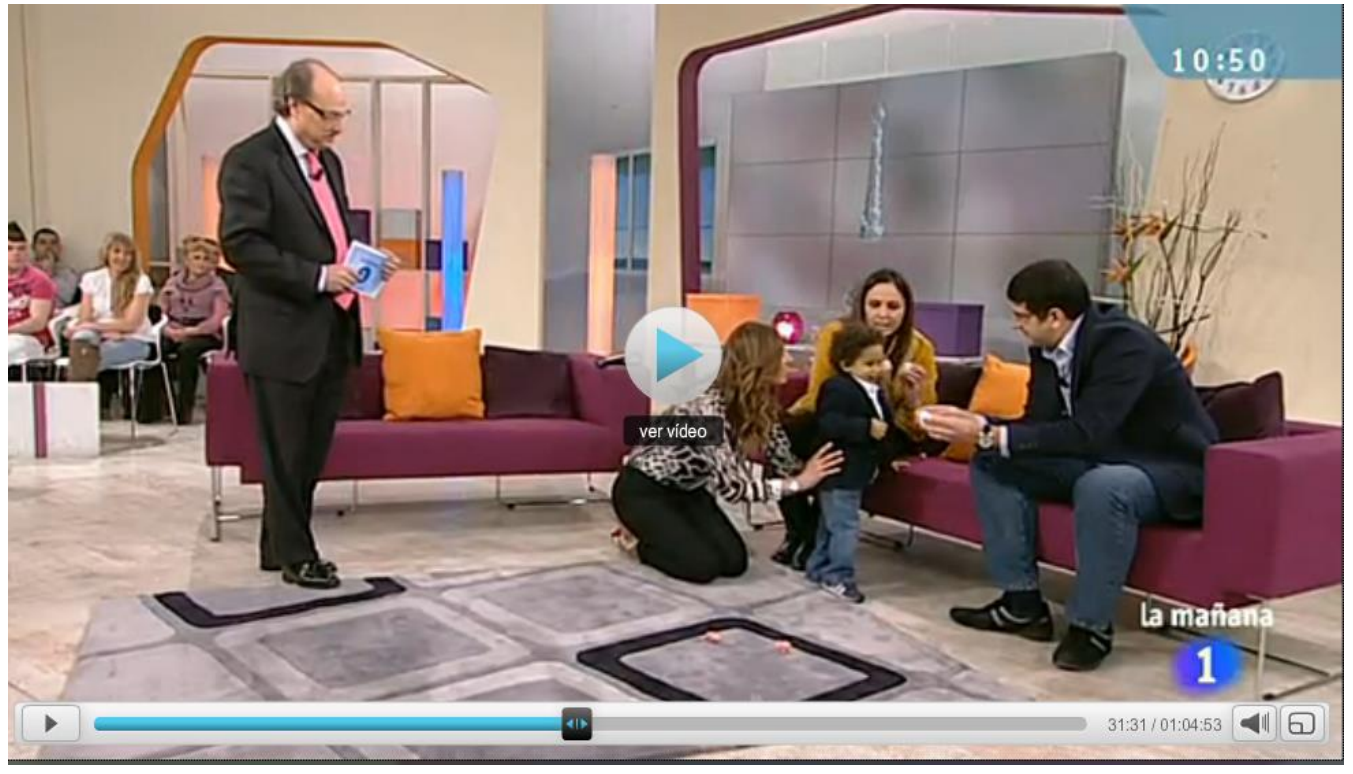

Fuente: http://www.rtve.es/alacarta/videos/la-manana/saber-vivir-29-02-12/1337081/ 
En el ámbito privado tienen lugar el $10 \%$ de las acciones que se desarrollan en estas noticias y reportajes, como el hogar cuando se trata de reportajes y noticias que tienen que ver con pacientes con alta dependencia, sobre todo niños. Hay un $13 \%$ de espacios que tienen lugar en ámbitos públicos y privados en la misma proporción.

\section{Conclusiones}

Para establecer las conclusiones fundamentales de esta investigación, damos respuesta a cada uno de los objetivos planteados en este estudio.

En relación al primer objetivo, establecemos que la cobertura en prensa ha sido mayor en el año 2013, el año de las patologías. Sin embargo, en radio y televisión a partir de 2013 comienza a aumentar y es en 2014 cuando se nota una consolidación y aumento de protagonismo de este colectivo. $A B C$ y la radiotelevisión pública, TVEy $R N E$, han otorgado mayor protagonismo a los pacientes y estas enfermedades. Se ha dedicado a la temática de estas patologías poco espacio tanto en prensa como en radio y televisión. Sin embargo, la radio ha profundizado más en la temática de las enfermedades raras a través de las entrevistas que ha realizado a los protagonistas de la información.

El hecho en sí de celebrar un Año Español de las Enfermedades Raras no ha sido relevante para la prensa, hay un tanto por ciento mínimo de información relacionada con el Año Español. Si que quizás se ha reforzado la presencia de personalidades que se han sumado a la causa, lo que ha potenciado el interés de los diarios, sobre todo en los encuadres visuales.

Con respecto al segundo objetivo, si comparamos los encuadres, teniendo en cuenta los frames que se generan en prensa tenemos que destacar el encuadre de interés humano. Se enfatiza una realidad social y, aunque se difunde también información científica, pesa más como realidad social en una situación problemática agravada por la crisis económica. Apreciamos cómo en todos los diarios hay un cambio de tendencia al referirse a las enfermedades raras, se pasa de resaltar el drama o la desatención a este colectivo a subrayar la actividad de las organizaciones de pacientes y científicos. El frame más destacado en los medios audiovisuales es también el de interés humano. Aunque el encuadre de movilización social tenga cierta ventaja en radio sobre el interés humano, siempre se relaciona con las historias personales de sus protagonistas. Mientras que en radio se centran en lo económico en 2013, y desciende este interés posteriormente, en televisión va en aumento porque se hacen más visibles los recortes que sufren los pacientes. Conforme avanzan los años, casi se duplica de 2012 a 2014 el número de noticias sobre problemas económicos que afectan a este colectivo.

La responsabilidad política queda en el aire, ya que se responsabiliza en general a las administraciones de la falta de atención, critican duramente el copago, la inexistencia de centros de referencia o la falta de recursos para posibles tratamientos, pero apenas se citan fuentes políticas concretas a las que reclamar esta desatención. Se podría decir que con respecto a las consecuencias económicas se sienten olvidados, solicitan mayor apoyo pero siempre lo buscan en la ciudadanía. El mensaje en radio es más neutral, más plano y objetivo, informativo, y en televisión el mensaje es testimonial relacionado con el interés humano. Los frames visuales en prensa y televisión ponen el foco en los pacientes como sujetos individuales y los periodistas utilizan de forma sistemática los encuadres analizados. En televisión se encuadran estos 
pacientes en espacios públicos, bien porque se graban o emiten en la vía pública, en centros sanitarios, congresos y por el aumento de actos organizados. El montaje de estos espacios es analítico, lo que significa que se ha buscado más la emotividad, planos cortos psicológicos y abundar en los detalles.

En relación al tercer objetivo, variación del tratamiento relacionando el Año Español de las Enfermedades Raras con el impacto mediático, hay que destacar que la Radiotelevisión española pública $R T V E$, tanto Radio Nacional, $R N E$, como Televisión Española, $T V E$, cumplen el compromiso que adquieren con las organizaciones de pacientes en 2013. A través de este análisis comprobamos que el cambio de tendencia con respecto al impacto en los medios se produce en 2013 y se consolida en 2014, ya que este año se produce la mitad de las piezas analizadas en radio y más de la mitad de las unidades analizadas en televisión. De esta investigación se deduce que la radio trata la temática de enfermedades raras con más frecuencia en programas, y la televisión dedica más espacio a estas patologías en los informativos. Por tanto, la duración en radio de estos espacios es mayor que en televisión. Los miembros de las organizaciones adquieren protagonismo en estas piezas con un papel de actores que reivindican una mejora social y mayor visibilidad del colectivo en la sociedad en 2013. Y en 2014 , son los científicos y médicos los que aparecen como protagonistas para reivindicar mayor atención a estas dolencias. Personajes con cierta relevancia social en los actos organizados son el reclamo que han utilizado estas organizaciones para llamar la atención de la prensa. La reina Doña Letizia acapara la mayoría de los titulares, a partir de 2013. La reina Doña Sofía, la periodista Isabel Gemio, el tenista Rafa Nadal, el seleccionador nacional Vicente del Bosque, son otras figuras de la vida social y cultural del país que aparecen en la prensa analizada.

Los medios de comunicación recurren como fuentes oficiales a los pacientes y los médicos. Los periodistas son responsables de poner el foco en estos colectivos y obtienen la información de quienes padecen la enfermedad y de los especialistas sanitarios que tratan dichas patologías. Por tanto, podemos dilucidar que, en este aspecto, la prensa ha recogido la información de quienes conocen de primera mano esta problemática. Las fuentes de las que se nutren estos medios audiovisuales son fundamentalmente los pacientes, de forma individual o mediante las organizaciones y familiares. Los profesionales de los medios de comunicación centran su atención en las personalidades se convierten en fuentes importantes para atraer a los medios en la mediante la cobertura de actos que las entidades organizan. La representación de los protagonistas de estos espacios suele ser a título individual tanto en radio como televisión. Esto se explica porque son fundamentales los pacientes junto a los miembros organizaciones $y$, aunque estos miembros representen al colectivo, normalmente suele resultar de interés el testimonio de las personas que padecen la enfermedad y, en muchas ocasiones, para contar lo que ocurre a un colectivo se recurre a una historia individual. Sin embargo, en radio se apela a los miembros de las organizaciones como portavoces y en televisión las fuentes son los pacientes a título personal, aunque le siguen de cerca los miembros de las organizaciones. Este hecho no varía a lo largo de los tres años analizados. Lo que si varía en radio es la presencia de pacientes, es mayor la representación de familiares en 2013 y 2014 que en 2012, esto se debe a que aumenta también la repercusión de enfermedades que afectan a niños y sus padres se erigen como abanderados en la causa. Los familiares organizan actos y movilizaciones para recaudar fondos. En televisión, sin embargo, son los pacientes los que se muestran a la opinión pública por encima de otros roles que tienen mayor presencia en el medio radiofónico.

Podríamos concluir que estas patologías están dejando de resultar raras a la población española y varía la representación de los pacientes. Predomina el frame de interés humano y movilización sobre el resto. Este 
es un hecho significativo, ya que de una imagen débil o una escasa representación en una fecha determinada, se ha pasado a representar a un colectivo que, aunque minoritario, cuenta con fuerza y apoyo suficiente para llevar a cabo iniciativas que les convierten en seres proactivos. Se podría afirmar con rotundidad que han logrado mayor visibilidad, pero aún están lejos de alcanzar sus objetivos en cuanto a la obtención de financiación por medio de la presencia en medios de comunicación. Por otra parte, las organizaciones de ER deben mejorar los procesos productivos de sus actividades dirigidas a los medios de comunicación, incluyendo sonido e imagen, puesto que son elementos esenciales en las rutinas productivas de estos medios, como es el caso específico de la radio y la televisión.

\section{Referencias bibliográficas}

Bañón Hernández, A. (2007). Las Enfermedades Raras y su representación discursiva. Propuestas para un análisis crítico. Discurso \& Sociedad, 1(2), pp. 188-229.

Bañón Hernández A.M., Alcaraz J.F., Almela J.S. \& Sanchis I.R. (2011). Desafíos y estrategias comunicativas de las enfermedades raras: la investigación médica como referente: estudio cuantitativo-discursivo de la prensa escrita impresa y digital española (2009-2010). Valencia: CIBERER, Centro de Investigación Biomédica en Red de Enfermedades Raras.

Castillo Esparcia, A., López Villafranca, P. \& Carretón Ballester, M.C. (2015). La comunicación en la red de pacientes con enfermedades raras en España. Revista Latina de comunicación social, (70), pp. 673688.

Entman, R. (1993). Framing: Toward a clarification of a fractured paradigm". Journal of Communication, 43(4), pp. 51-58.

Igartua, J. J., \& Humanes, M. L. (2004). Imágenes de Latinoamérica en la prensa española. Una aproximación empírica desde la Teoría del Encuadre. Comunicación y Sociedad, 171), pp 47-75.

Liu, B. F. \& Kim, S. (2011). How organizations framed the $2009 \mathrm{H} 1 \mathrm{~N} 1$ pandemic via social and traditional media: Implications for US health communicators. Public Relations Review, 373), pp. 233-244.

McCombs, M. E., \& Shaw, D. L. (1993). The evolution of agenda-setting research: twenty-five years in the marketplace of ideas. Journal of communication, 43(2), pp. 58-67.

Piñuel, J. (1999). Formación universitaria y formación de profesionales en Métodos y Técnicas de Investigación Social en Comunicación. Madrid: Universidad Complutense de Madrid.

Posada de la Paz, M. (2008). Las enfermedades raras y su impacto en la gestión de los servicios de salud. Revista de administración sanitaria siglo XXI , 6(1), pp. 157-178.

Rodero, E., Maíllo A. P. \& Tamarit, A. (2009). El atentado del 11 de marzo de 2004 en la Cadena SER desde la teoría del 'framing'. Zer-Revista de Estudios de Comunicación, (26), pp. 81-103.

Sánchez Castillo, S. (2012). Representación social de las enfermedades raras en la prensa española. Aposta: Revista de ciencias sociales, (54), p. 1.

Sánchez Castillo, S. (2013) Las enfermedades raras en la prensa española: una aproximación empírica desde la teoría del framing. Ambitos: Revista internacional de comunicación, (22), pp. 71-80.

Sánchez Castillo, S. \& Mercado, M.T. (2014). El encuadre de las enfermedades raras en la prensa española. Anuario electrónico de estudios en Comunicación Social. Disertaciones, $\nearrow 1$ ), pp. 104-128. 
Semetko, H. A. \& Valkenburg, P. M. (2000). Framing European politics: A content analysis of press and television news. Journal of communication, 50(2), pp. 93-109.

Urrutia, J.M. (2013). El Día Mundial de las Enfermedades Raras en las noticias de prensa. El ejemplo de Andalucía. Discurso \& Sociedad, 71 , pp. 224-247.

Villa, À. À., Llombart, B. E., Solanillo, C. F., Queralt, A. R. \& Castillo, S. S. (2014). Las Enfermedades Raras en los Medios. Madrid: Observatorio de Enfermedades Raras, OBSER. 